\title{
eHealth: die Rechte der Patienten und der Ärzteschaft schützen
}

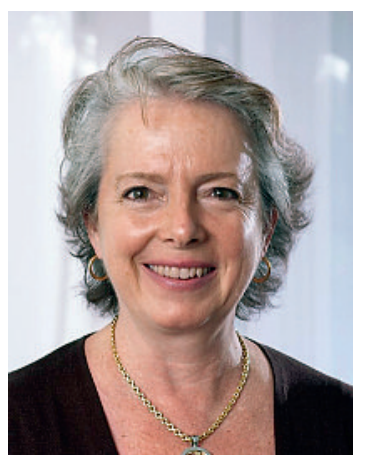

Bereits 12000 Health Professional Cards (FMH-HPC) hat die FMH bis heute abgegeben. Dies belegt, dass wir eine der Rollen, die von einer Standesorganisation erwartet werden dürfen, perfekt erfüllen: das Bereitstellen einer Chipkarte, die einen abgesicherten, eindeutigen Nachweis der Qualifikation im Internet ermöglicht. Die von uns entwickelte, offene Karte - auf die wir stolz sein dürfen! - ist intelligent konzipiert und entspricht sowohl den schweizerischen wie auch den europäischen Standards. Sie kann auch als Träger für weitere Zertifikate dienen, die andere Bereiche abdecken - zum Beispiel jene der SuisseID: Die beiden Zertifikatangebote ergänzen sich gegenseitig.

Mit der FMH-HPC verfügen wir über ein zentrales Teil des eHealth-Puzzles, denn mit ihr können wir sowohl unsere Identität als auch unsere Qualifikation als Ärztinnen und Ärzte nachweisen: Sie sollte den Zugangsschlüssel für alle Applikationen bilden, die sich an die Ärzteschaft richten.
Es ist wichtig, dass wir den Bereich eHealth aktiv gestalten, indem wir die gewünschte Richtung vorgeben und standardisierte Systeme verlangen, die zwingend kompatible Schnittstellen aufweisen. Dass dies nicht einfach ist, zeigen

\section{Mit der FMH-HPC verfügen wir über ein zentrales Teil des eHealth-Puzzles}

die Schwierigkeiten, die wir heute mit der von den Krankenkassen entwickelten Versichertenkarte haben. Und sie zeigen auch, dass wir nicht von den grundlegenden Regeln abweichen dürfen. Die für den Zugriff auf medizinische Daten auf der Versichertenkarte erforderliche Kommunikation zwischen der FMH-HPC und der Versichertenkarte ist heute noch immer problematisch; ihr Nutzen bleibt für die Ärzteschaft weiterhin sehr fraglich.

Sieht man sich im Ausland um, wurden die besten aktuellen Standards im Gesundheitsbereich, wie zum Beispiel HL7, oft von der Ärzteschaft entwickelt. Es liegt an uns, die sich

\section{Es ist wichtig, dass wir den Bereich eHealth aktiv gestalten, indem wir die gewünschte Richtung vorgeben und standardisierte Systeme verlangen}

Auch sollten wir der Einführung anderer Karten widerstehen, wenn wir verhindern möchten, dass Nischen mit proprietären Systemen und unvorhergesehenen zusätzlichen Kosten entstehen, bei denen uns die Hände gebunden sind.

Die einzelnen Teile des Puzzles werden derzeit entwickelt. Die Ärzteschaft legt vor allem Wert darauf, dass die Gesundheitsapplikationen benutzerfreundlich sind*, gemäss der Philosophie, die zunächst bei den Palms zum Einsatz gelangte und auf der auch das iPhone beruht: Die Benutzerin oder der Benutzer erkundet die verfügbaren Anwendungen und kann sich nach dem Prinzip «Learning by doing» mit ihnen vertraut machen. abzeichnenden Entwicklungen im Auge zu behalten und Lösungen einzubringen, die sich als optimale Basis für eHealth in der Schweiz anbieten.

Monique Gauthey, Fachärztin FMH, Mitglied des Zentralvorstands der FMH, Verantwortliche für das Ressort eHealth

* vgl. Strategische Ziele «eHealth» der FMH, www.fmh.ch $\rightarrow$ Weitere Themen $\rightarrow$ eHealth $\rightarrow$ eHealth-Strategien 